\title{
Anomalous Hall Effect in MnAl/W bilayers: Modification from strong spin Hall effect of W
}

\author{
K. K. Meng ${ }^{1,}{ }^{*}$, J. Miao ${ }^{1}$, X. G. Xu ${ }^{1}$, Y. Wu ${ }^{1}$, J. H. Zhao ${ }^{2}$ and Y. Jiang ${ }^{1}$ \\ ${ }^{1}$ School of Materials Science and Engineering, University of Science and \\ Technology Beijing, Beijing 100083, China \\ ${ }^{2}$ State Key Laboratory of Superlattices and Microstructures, Institute of \\ Semiconductors, Chinese Academy of Sciences, Beijing 100083, China
}

\begin{abstract}
We report systematic measurements of anomalous Hall effect (AHE) in MnAl/W bilayers modified by strong spin Hall effect (SHE) of the heavy metals, in which a single $L 1_{0}-\mathrm{MnAl}$ epitaxial layer reveals obvious orbital two-channel Kondo (2CK) effect. The results are compared with the $\mathrm{AHE}$ in $\mathrm{MnAl} / \mathrm{Cu}$ with weak spin orbit coupling. As increasing the thickness of $\mathrm{W}$, the strong SHE has gradually suppressed the orbital $2 \mathrm{CK}$ effect and modified the AHE of MnAl. A scaling involving multiple competing scattering mechanisms has been used to distinguish different contributions to the modified AHE. The direct observation of spin-orbit torque induced magnetization switching confirms that the result is a combination of the AHE of MnAl and SHE of W.
\end{abstract}

Keywords: Spin transport through interfaces; Spin-orbit coupling; Magnetoelectronics

PACS: 72.25.Mk; 71.70.Ej; 85. 75.-d

*Authors to whom correspondence should be addressed:

kkmeng@ustb.edu.cn 


\section{Introduction}

The anomalous Hall effect (AHE) is the most prominent phenomenon that exists in ferromagnetic materials (FM), which originates from the interplay between spin orbit coupling (SOC) and magnetism [1]. Electrons moving through a FM will acquire a transverse velocity with opposite directions for different spin orientations due to SOC, since the charge currents have usually a net polarization, this spin-dependent transverse velocity will result in a net transverse anomalous Hall voltage [2]. Recent investigations have both theoretically and experimentally explored the possible mechanisms of AHE through giving a unified scaling describing the AHE resistivity $\rho_{\mathrm{AH}}$ in terms of the longitudinal resistivity $\rho_{\mathrm{xx}}$. Karplus and Luttinger have proposed that the intrinsic AHE arises from the transverse velocity of Bloch electrons induced by SOC together with interband mixing, giving $\rho_{\mathrm{AH}} \sim \rho_{\mathrm{xx}}^{2}[3-8]$. On the other hand, the extrinsic mechanisms including skew scattering and side jump come from the asymmetrical scattering of conduction electrons due to SOC, giving $\rho_{\mathrm{AH}} \sim \rho_{\mathrm{xx}}$ and $\rho_{\mathrm{AH}} \sim \rho_{\mathrm{xx}}^{2}$, respectively [9-11]. Notably, in contrast to the conventional picture that $\rho_{\mathrm{AH}}$ scales with the total resistivity irrespective of its sources [12], recent experimental studies have revealed that both $\rho_{\mathrm{AH}}$ and the scaling relation are qualitatively different for various types of electron scattering. Working on epitaxial GaAs/Fe films, Tian et al have limited the scattering of electrons to two sources, one by interface roughness and another by phonons, with independent control on their strengths through the film thickness and sample temperature [13]. They developed the experimental strategy neglecting the contribution of phonon skew scattering, giving the scaling $\rho_{\mathrm{AH}}=a_{0} \rho_{\mathrm{xx} 0}+b \rho_{\mathrm{xx}}^{2}=k_{0} \rho_{\mathrm{xx} 0}+\beta \rho_{\mathrm{xx} 0}^{2}+b \rho_{\mathrm{xx}}^{2}$, where $\rho_{\mathrm{xx} 0}$ is the residual resistivity induced by impurity scattering. However, most recently, Hou et al have derived a general scaling relation for the AHE in FM involving multiple competing scattering mechanisms, described by a quadratic hypersurface in the space spanned by the partial resistivities, giving [14]:

$$
\rho_{\mathrm{AH}}=\alpha \rho_{\mathrm{xx} 0}+\beta_{0} \rho_{\mathrm{xx} 0}^{2}+\gamma \rho_{\mathrm{xx} 0} \rho_{\mathrm{xxT}}+\beta_{1} \rho^{2}{ }_{\mathrm{xxT}}
$$

where $\rho_{\mathrm{xx} 0}$ is the residual resistivity induced by impurity scattering, $\rho_{\mathrm{xxT}}=\rho_{\mathrm{xx}}-\rho_{\mathrm{xx} 0}$ is 
due to dynamic disorders (mainly phonons at higher temperatures), $\alpha$ is the skew scattering coefficient, the coefficients $\beta_{0}, \beta_{1}$ and $\gamma$ contain intrinsic Berry curvature and side-jump contributions. Spin Hall effect (SHE) that converts charge currents into spin currents in a heavy metal (HM) with strong SOC has attracted much interest due to its practical use in technological applications [15-22]. In these hybrid structures, spin and charge transport phenomena are interconnected, and HM may serve as both spin current generator and detector. The spin currents that diffuse into the FM will modify the spin dependent transport properties such as AHE [23]. On the other hand, the spin currents will also exert torque on the magnetization, which is similar to spin transfer torque [24-31]. Considering the essence of AHE, the modification from SHE can also be explored through clarifying $\rho_{\mathrm{AH}} \sim \rho_{\mathrm{xx}}$ relations [23].

Another spin-dependent transport behavior that can dramatically influence the AHE is the orbital two-channel Kondo (2CK) effect [32]. When a pseudo-spin 1/2 of structural two-level system strongly couples to two spin channels of conduction electrons, the orbital $2 \mathrm{CK}$ effect will become evident with displaying exotic non-Fermi liquid (NFL) behavior [33]. The 2CK effect has a unique temperature (T) dependence in resistivity with three different $\mathrm{T}$ ranges: a low $\mathrm{T}$ upturn $\Delta \rho_{\mathrm{xx}}$, which scales with $\ln \mathrm{T}$ beyond the Kondo temperature $\mathrm{T}_{\mathrm{K}}$, followed by NFL behavior $\Delta \rho_{\mathrm{xx}} \sim \mathrm{T}^{1 / 2}$ for $\mathrm{T}_{\mathrm{D}}<\mathrm{T}<\mathrm{T}_{\mathrm{K}}$ as the consequence of two conduction electron spins screening, then at extreme low temperature a deviation from $\mathrm{T}^{1 / 2}$ dependence has happened [32-34]. The most remarkable characteristic in the orbital $2 \mathrm{CK}$ effect is the NFL behavior $\Delta \rho_{\mathrm{xx}} \sim \mathrm{T}^{1 / 2}$, which is much different from the $\Delta \rho_{\mathrm{xx}} \sim \mathrm{T}^{2}$ scaling of Fermi-liquid behavior in fully screened Kondo effect. Recently, Zhu et al. have reported that the AHE in perpendicularly magnetized MnAl epitaxial films with a variable orbital $2 \mathrm{CK}$ effect [32]. The AHE is observed to excellently scale with $\rho_{\mathrm{AH}} / f=a_{0} \rho_{\mathrm{xx} 0}+b \rho_{\mathrm{xx}}^{2}$ at high temperatures. However, a significant deviation happens at low temperatures where the orbital $2 \mathrm{CK}$ effect becomes important, which agrees with the orbital $2 \mathrm{CK}$ effect in the breakdown temperatures and deviation magnitudes.

In this context, as an attempt to offer a step towards exploring these rich and underlying physics, we have systematically investigated the AHE in MnAl/W films, 
in which a single $L 1_{0}-\mathrm{MnAl}$ epitaxial layer reveals obvious orbital $2 \mathrm{CK}$ effect. The strong SHE of $\mathrm{W}$ has suppressed the orbital 2CK effect and modified the AHE of MnAl. The results are compared with those in $\mathrm{MnAl} / \mathrm{Cu}(6 \mathrm{~nm})$ bilayers, in which $\mathrm{Cu}$ has very weak SOC. It is found that the spin-dependent transport properties in $\mathrm{MnAl} / \mathrm{W}(\mathrm{t})$ ( $\mathrm{t}$ is in nanometre) are much different from that in the MnAl single layer and the $\mathrm{MnAl} / \mathrm{Cu}$ films. Using the scaling developed by Hou et al, different contributions to the modified $\mathrm{AHE}$ in $\mathrm{MnAl}, \mathrm{MnAl} / \mathrm{Cu}$ and $\mathrm{MnAl} / \mathrm{W}$ can be distinguished [14]. The scaling analysis reveals that the most evident modification happened in the coefficients $\gamma$ and $\beta_{1}$. The modified AHE is a combination of the AHE in $\mathrm{MnAl}$ and SHE in $\mathrm{W}$, which is proved by the measurement of SOT.

\section{Experimental details}

A 4-nm-thick $L 1_{0}-\mathrm{MnAl}$ single-crystalline film was grown on a semi-insulating GaAs (001) substrate by molecular-beam epitaxy at substrate temperature $T_{\mathrm{S}}=473 \mathrm{~K}$. Here the $\mathrm{Mn} / \mathrm{Al}$ atoms ratio is 1 . Then, $\mathrm{Cu}$, and $\mathrm{W}$ films with different thickness $\mathrm{t}=2,4$, $6 \mathrm{~nm}$ were deposited on it by dc magnetron sputtering immediately. Photolithography and Ar ion milling were used to pattern Hall bars and a lift-off process was used to form the contact electrodes. The size of all the Hall bars is $10 \mu \mathrm{m} \times 80 \mu \mathrm{m}$. The transport measurements were carried out in a physical property measurement system (PPMS). We have also measured the temperature dependent resistivities of single $\mathrm{Cu}$ and $\mathrm{W}$ films. Then, assuming that each film in the $\mathrm{MnAl} / \mathrm{Cu}$ and $\mathrm{MnAl} / \mathrm{W}$ bilayers acts as a parallel resistance path, both the AHE resistivity $\rho_{\mathrm{AH}}$ and the longitudinal resistivity $\rho_{\mathrm{xx}}$ in this manuscript have been expressed as those of the MnAl layer.

\section{Results and discussion}

The normalized out-of-plane hysteresis loops of $\mathrm{MnAl}$ film is shown in Figure 1(a). The perpendicular $\mathrm{M}-\mathrm{H}$ curve shows a square-like shape revealing its giant perpendicular magnetic anisotropy (PMA). Fig. 1 (b) plot the T dependence of the resistivity variation at $\mathrm{H}=0$ Oe and $\mathrm{H}=80000$ Oe for the $L 1_{0}-\mathrm{MnAl}$ film, the $\rho_{\mathrm{xx}}$ under these two magnetic field all show an increase arising from the orbital $2 \mathrm{CK}$ effect. Fig. 1 (c) and (d) have shown distinct characteristic, which is a typical transport behavior of orbital $2 \mathrm{CK}$ effect. The longitudinal resistivity $\rho_{\mathrm{XX}}$ firstly varies linearly with $\ln \mathrm{T}$ 
as increasing $\mathrm{T}$ from $300 \mathrm{~K}$. Then, $\rho_{\mathrm{XX}}$ deviates from the $\ln \mathrm{T}$ dependence and turns to a $T^{1 / 2}$ dependence when $T$ is below $T_{K}=105 K$. The large $T_{K}$ of the $L 1_{0}-\mathrm{MnAl}$ film suggests a strong coupling between a pseudo-spin $1 / 2$ of structural two-level system and the two spin channels of conduction electrons. The $\mathrm{T}^{1 / 2}$-dependent resistivity is a distinguishing feature of the NFL behavior for the 2CK effect. As further decreasing temperature, $\rho_{\mathrm{XX}}$ begins to increase faster than $\mathrm{T}^{1 / 2}$ below a characteristic temperature $\mathrm{T}_{\mathrm{D}}=40 \mathrm{~K}$. On the other hand, magnetic fields $\mathrm{H}$ should not have any observable influence on the orbital $2 \mathrm{CK}$ effect via changing the population balance of the two spin channels of the conduction electrons. The H-independent resistivity upturn as shown in Fig. 1(a) scaling with $\ln \mathrm{T}$ and $\mathrm{T}^{1 / 2}$ in the two $\mathrm{T}$ regimes, and the deviation from the NFL behavior at the lowest temperatures are consistent with the transport properties in two-level systems [32, 33].

After depositing a 6-nm-thick $\mathrm{Cu}$ film, the orbital 2CK effect has not been changed evidently, as shown in Fig. 2(a). Notably, comparing the T dependent of $\rho_{\mathrm{XX}}$ in $\mathrm{MnAl}$ and $\mathrm{MnAl} / \mathrm{Cu}$, evident suppression has been found in $\mathrm{MnAl} / \mathrm{W}(4 \mathrm{~nm})$ and $\mathrm{MnAl} / \mathrm{W}(6 \mathrm{~nm})$ as shown in Fig. 2(c) and (d). At a characteristic temperature $\left(\mathrm{T}_{\mathrm{M}}=90\right.$ $\mathrm{K})$, there is a resistivity minimum of $\rho_{\mathrm{XX}}$ for the $\mathrm{MnAl} / \mathrm{W}(4 \mathrm{~nm})$ bilayer. In the high $\mathrm{T}$ range $\left(\mathrm{T}>\mathrm{T}_{\mathrm{M}}\right), \rho_{\mathrm{XX}}$ increases linearly with $\mathrm{T}$ due to the increasing phonon scattering, and $T_{K}$ decreases to $50 \mathrm{~K}$. The $\mathrm{T}_{K}$ and $\mathrm{T}_{\mathrm{D}}$ of all the films have been evaluated from Fig. 1 and 3. The suppression gradually increased as increasing the thickness of $\mathrm{W}$ with further decreasing $T_{K}$ and $T_{D}$ as summarized in Table I. Notably, as discussed by Zhu et al, the ferromagnetic exchange splitting of the $d$-band will lead to an imbalance in the channel population and quench the NFL behavior, which is characterized by an enhanced effective breakdown temperature of the 2CK effect [33]. The channel asymmetry should lead to different tunnelling rates of the two-level system for two spin channels and thus weaken the NFL behaviors in comparison with its nonmagnetic counterpart $\left(\Delta \mathrm{N}=\mathrm{N}_{\uparrow}-\mathrm{N}_{\downarrow}=0\right)$, where $\mathrm{N}_{\uparrow}$ and $\mathrm{N}_{\downarrow}$ are the density of states for majority and minority spin channels in the conduction band. Based on the Stoner model for itinerant ferromagnetism, the saturation magnetization $\mathrm{M}_{\mathrm{S}}$ can be used as an index for the degree of spin population imbalance of the conduction band. 
The small $\mathrm{M}_{\mathrm{S}}$ of the $\mathrm{MnAl}$ film studied here indicates a very low degree of spin population imbalance with the loss of the chemical ordering. This could be the reason why the ferromagnetic exchange splitting does not quench the 2CK physics [31]. Considering the enhanced channel asymmetry will weaken the NFL behavior, we suppose that the orbital $2 \mathrm{CK}$ effect will be suppressed if the pure spin current generated by the metals with strong SOC, such as W, diffuses into the FM. The spin polarization at the Fermi surface will be modified and the enhanced $\Delta \mathrm{N}$ will quench the orbital $2 \mathrm{CK}$ effect.

Fig. 4(a) shows the T profiles of $\rho_{\mathrm{AH}}$ for all the five samples, which all decrease as cooling down. So far, there have been few reports on a similar anomaly in the $\mathrm{T}$ dependence of $\rho_{\mathrm{AH}}[35]$. We infer that the strong orbital $2 \mathrm{CK}$ effect in $L 1_{0}-\mathrm{MnAl}$ could be the reason for such a complex T dependence of $\rho_{\mathrm{AH}}$. We plot $\rho_{\mathrm{AH}}$ as a function of $\rho_{\mathrm{xx}}$ for all the samples to compare the difference of scaling curves in Fig. 4 (b). It is found that the function of $\rho_{\mathrm{AH}}\left(\rho_{\mathrm{xx}}\right)$ in $\mathrm{MnAl}$ is much different with $\mathrm{MnAl} / \mathrm{W}$. Moreover, varying the thickness of $\mathrm{W}$ also changes the function of $\rho_{\mathrm{AH}}\left(\rho_{\mathrm{xx}}\right)$. Considering that the conductivity of ultrathin $\mathrm{MnAl}$ film is small enough, we firstly use the scaling given by Tian et al for describing the AHE in this system [13]. In Fig. $4(\mathrm{c})$, the $\rho_{\mathrm{AH}}$ is plotted versus the longitudinal resistivity square $\rho_{\mathrm{XX}}^{2}$. However, we found that for all the films these plots have not shown linearity from high temperature to low temperature, indicating that this scaling cannot work. It is indicated that the intrinsic anomalous Hall conductivity is temperature dependent, which can be ascribed to the orbital $2 \mathrm{CK}$ effect.

Then, using Equation (1), we have taken experimental data of $\rho_{\mathrm{AH}}\left(\rho_{\mathrm{xx}}\right)$ and fitted curves in $\mathrm{MnAl}, \mathrm{MnAl} / \mathrm{W}(4 \mathrm{~nm})$ and $\mathrm{MnAl} / \mathrm{W}(6 \mathrm{~nm})$ as examples as shown in Fig. 5. It is found that the scaling works well for $\mathrm{MnAl}$ at the whole range of temperature. A deviation happened at low temperatures in $\mathrm{MnAl} / \mathrm{W}(6 \mathrm{~nm})$, where the orbital $2 \mathrm{CK}$ effect becomes important. For MnAl/W(4nm), the scaling has been used at different range of temperature $\mathrm{T}<90 \mathrm{~K}$ and $\mathrm{T}>90 \mathrm{~K}$ respectively, and different coefficients have been deduced as shown in Table II. The most evident modification happened in the coefficients $\gamma$ and $\beta_{1}$. As discussed by Hou et al., it is really hard to 
separate out the intrinsic part from the side-jump one in $\beta_{1}$ and $\gamma$ [14]. However, $\left(\beta_{0}+\beta_{1}-\gamma\right)$ and $\left(\gamma-2 \beta_{1}\right)$ are the coefficients of pure scattering effect and they have also changed sign with thick HM ( $>4 \mathrm{~nm})$. Given that only the four parameters (and their linear combinations) can be extracted, it seems challenging to unambiguously separate out the intrinsic contribution from such experimental approach. This will surely motivate further experimental studies in near future. Considering the chemical ordering of $\mathrm{MnAl}$ has not been changed, the variation of extrinsic contribution should partly come from the asymmetric scattering due to the effective SOC at the interface and partly come from the modification from injected spin-polarized current.

To further verify our proposition, we have carried out the SHE induced switching of perpendicular magnetization in for $\mathrm{MnAl} / \mathrm{W}(6 \mathrm{~nm})$. The $\rho_{\mathrm{AH}}$ was measured as a function of perpendicular magnetic field under different input currents $(1,8,12 \mathrm{~mA})$ as shown in Fig. 6. When the input current is as small as $1 \mathrm{~mA}$, the coercivity of $\mathrm{MnAl} / \mathrm{W}(6 \mathrm{~nm})$ is about 10000 Oe. As the current is increased to $12 \mathrm{~mA}$, the coercivity dramatically decreases to 6000 Oe. On the other hand, $\rho_{\mathrm{AH}}$ has also decreased. We believe it is due to the increase of current-induced torque related with SHE as discussed $\mathrm{MnGa} / \mathrm{Ta}$ films [23]. However, the current-induced torque has not been found in $\mathrm{MnAl} / \mathrm{Cu}$ films. Thus it further confirms that the modified AHE discussed above stems from the SHE in the heavy metals, and the result is the combination of the AHE of MnAl and the SHE of the heavy metals.

\section{Summary}

In summary, we have systematically investigated $\mathrm{AHE}$ in $\mathrm{MnAl} / \mathrm{W}$ bilayers modified by strong SHE of the heavy metals, in which a single $L 1_{0}$-MnAl epitaxial layer reveals obvious orbital $2 \mathrm{CK}$ effect. The results are compared with the AHE in $\mathrm{MnAl} / \mathrm{Cu}$ with weak spin orbit coupling. As increasing the thickness of $\mathrm{W}$, the strong SHE has gradually suppressed the orbital $2 \mathrm{CK}$ effect and modified the AHE of MnAl. A scaling involving multiple competing scattering mechanisms has been used to distinguish different contributions to the modified AHE. The scaling analysis reveals that the most evident modification happened in the coefficients $\gamma$ and $\beta_{1}$. The direct observation of SOT induced magnetization switching confirms that the result is a 
combination of the AHE of MnAl and SHE of W.

This work was partially supported by the National Basic Research Program of China (2015CB921502), the National Science Foundation of China (Grant Nos. 61404125, 51371024, 51325101, 51271020).

\section{References}

[1] E. H. Hall, Philos. Mag. 10 (1880) 301.

[2] A. Hoffmann, IEEE Trans. Magn. 49 (2013) 5172.

[3] R. Karplus and J. M. Luttinger, Phys. Rev. 95 (1954) 1154.

[4] G. Sundaram and Q. Niu, Phys. Rev. B 59 (1999) 14915.

[5] T. Jungwirth, Q. Niu, and A. H. MacDonald, Phys. Rev. Lett. 88 (2002) 207208.

[6] M. Onoda and N. Nagaosa, J. Phys. Soc. Jpn. 71 (2002) 19.

[7] J. Sinova, D. Culcer, Q. Niu, N. A. Sinitsyn, T. Jungwirth, and A. H. MacDonald, Phys. Rev. Lett. 92 (2004) 126603.

[8] D. Xiao, M.-C. Chang, and Q. Niu, Rev. Mod. Phys. 82 (2010) 1959.

[9] J. Smit, Physica (Amsterdam) 21 (1955) 877.

[10] L. Berger, Phys. Rev. B 2 (1970) 4559.

[11] A. Crepieux and P. Bruno, Phys. Rev. B 64 (2001) 014416.

[12] P. He, L. Ma, Z. Shi, G. Y. Guo, J. G. Zheng, Y. Xin, and S. M. Zhou, Phys. Rev. Lett. 109 (2012) 066402.

[13] Y. Tian, L. Ye, and X. Jin, Phys. Rev. Lett. 103 (2009) 087206.

[14] D. Z. Hou, G. Su, Y. Tian, X. F. Jin, S. Y. A. Yang, Q. Niu, Phys. Rev. Lett. 114 (2015) 217203.

[15] J. E. Hirsch, Phys. Rev. Lett. 83 (1999) 1834.

[16] I. M. Miron, K. Garello, G. Gaudin, P. J. Zermatten, M. V. Costache, S. Auffret, S. Bandiera, B. Rodmacq, A. Schuhl, and P. Gambardella, Nature 476 (2011) 189.

[17] L. Q. Liu, C.-F. Pai, Y. Li, H. W. Tseng, D. C. Ralph, and R. A. Buhrman, Science 336 (2012) 555.

[18] K. Garello, I. M. Miron, C. O. Avci, F. Freimuth, Y. Mokrousov, S. Blügel, S. 
Auffret, O. Boulle, G. Gaudin, and P. Gambardella, Nat. Nanotechnol. 8 (2013) 587.

[19] H. Nakayama, M. Althammer, Y.-T. Chen, K. Uchida, Y. Kajiwara, D. Kikuchi, T.

Ohtani, S. Geprägs, M. Opel, S. Takahashi, R. Gross, G. E. W. Bauer, S. T. B. Goennenwein, and E. Saitoh, Phys. Rev. Lett. 110 (2013) 206601.

[20] J. Kim, J. Sinha, M. Hayashi, M. Yamanouchi, S. Fukami, T. Suzuki, S. Mitani, and H. Ohno, Nat. Mater. 12 (2013) 240.

[21] L. Q. Liu, O. J. Lee, T. J. Gudmundsen, D. C. Ralph, and R. A. Buhrman, Phys. Rev. Lett. 109 (2012) 096602.

[22] S. Emori, U. Bauer, S.-M. Ahn, E. Martinez, and G. S. D. Beach, Nat. Mater. 12 (2013) 611.

[23] K. K. Meng, J. Miao, X. G. Xu, J. X. Xiao, J. H. Zhao, and Y. Jiang, Phys. Rev. B 93 (2016) 060406(R).

[24] C. O. Avci, K. Garello, C. Nistor, S. Godey, B. Ballesteros, A. Mugarza, A. Barla, M. Valvidares, E. Pellegrin, A. Ghosh, I. M. Miron, O. Boulle, S. Auffret, G. Gaudin, and P. Gambardella, Phys. Rev. B 89 (2014) 214419.

[25] G. Yu, P. Upadhyaya, Y. Fan, J. G. Alzate, W. Jiang, K. L. Wong, S. Takei, S. A. Bender, L.-T. Chang, Y. Jiang, M. Lang, J. Tang, Y. Wang, Y. Tserkovnyak, P. K. Amiri, and K. L. Wang, Nat. Nanotechnol. 9 (2014) 548.

[26] C. Zhang, S. Fukami, H. Sato, F. Matsukura, and H. Ohno, Appl. Phys. Lett. 107 (2015) 012401.

[27] S. Fukami, C. L. Zhang, S. DuttaGupta, A. Kurenkov, and H. Ohno, Nat. Mater. 15 (2016) 4.

[28] T. Suzuki, S. Fukami, N. Ishiwata, M. Yamanouchi, S. Ikeda, N. Kasai, and H. Ohno, Appl. Phys. Lett. 98 (2011) 142505.

[29] X. Qiu, K. Narayanapillai, Y. Wu, P. Deorani, D.-H. Yang, W.-S. Noh, J.-H. Park, K.-J. Lee, H.-W. Lee, and H. Yang, Nat. Nanotechnol. 10 (2015) 333.

[30] X. Qiu, P. Deorani, K. Narayanapillai, K.-S. Lee, K.-J. Lee, H.-W. Lee, and H. Yang, Sci. Rep. 4 (2014) 4491.

[31] K. K. Meng, J. Miao, X. G. Xu, Y, Wu, J. X. Xiao, J. H. Zhao, and Y. Jiang, Sci. Rep. 6 (2016) 38375. 
[32] L. J. Zhu, S. H. Nie, and J. H. Zhao, Phys. Rev. B 93 (2016) 195112.

[33] L. J. Zhu, S. H. Nie, P. Xiong, P. Schlottmann, and J. H. Zhao, Nat. Commun. 7 (2016) 10817.

[34] D. L. Cox and A. Zawadowki, Adv. Phys. 47 (1998) 599.

[35] W. Zhang, M. B. Jungfleisch, W. Jiang, J. E. Pearson, A. Hoffmann, F. Freimuth, and Y. Mokrousov, Phys. Rev. Lett. 113 (2014) 196602.

\section{Figure Captions}
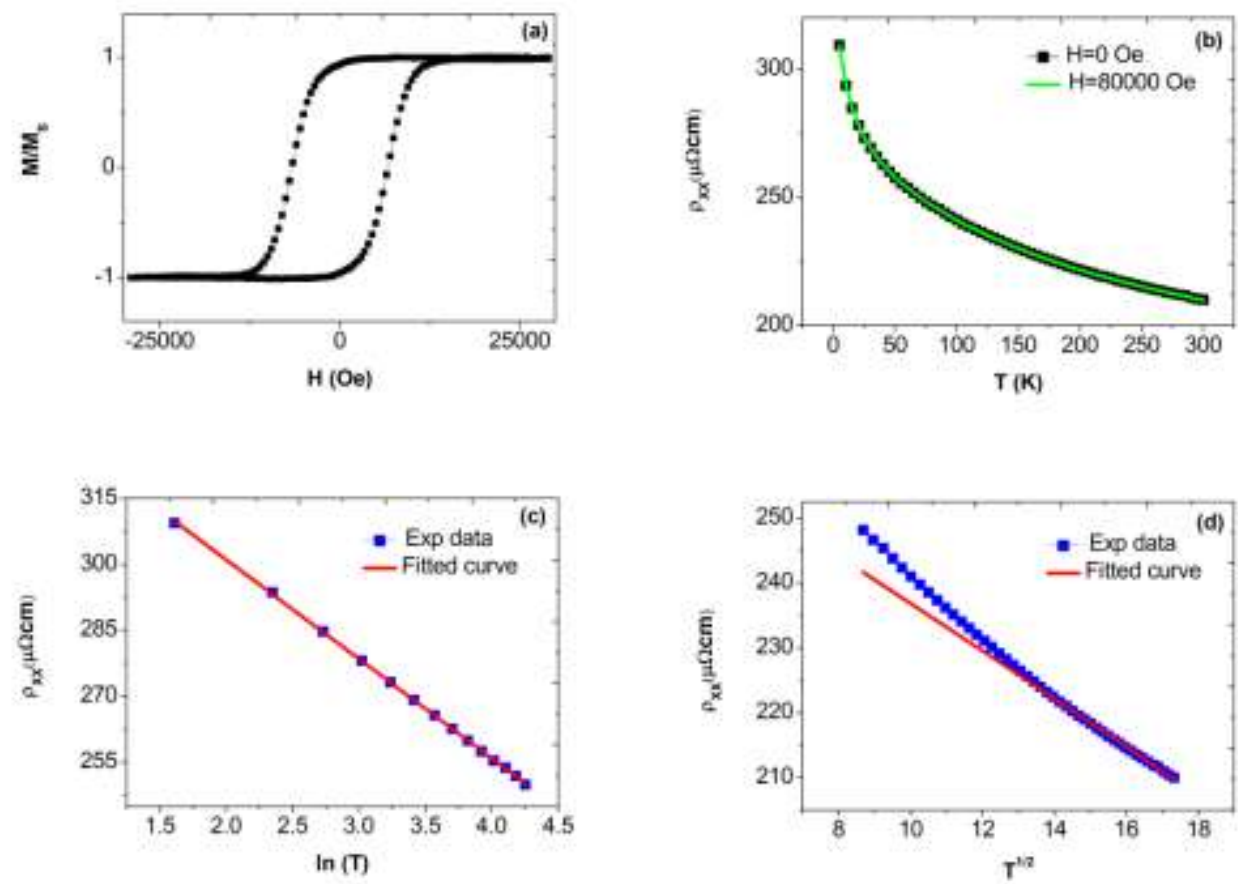

Fig. 1 (a) The normalized out-of-plane hysteresis loop of MnAl film. (b) $\rho_{\mathrm{xx}} \mathrm{vs} \mathrm{T}$ at $\mathrm{H}=0$ Oe (a) and $\mathrm{H}=80000$ Oe for MnAl film. (c) and (d) $\rho_{\mathrm{xx}} \mathrm{vs} \mathrm{T}$ for MnAl film in different range of temperature. 

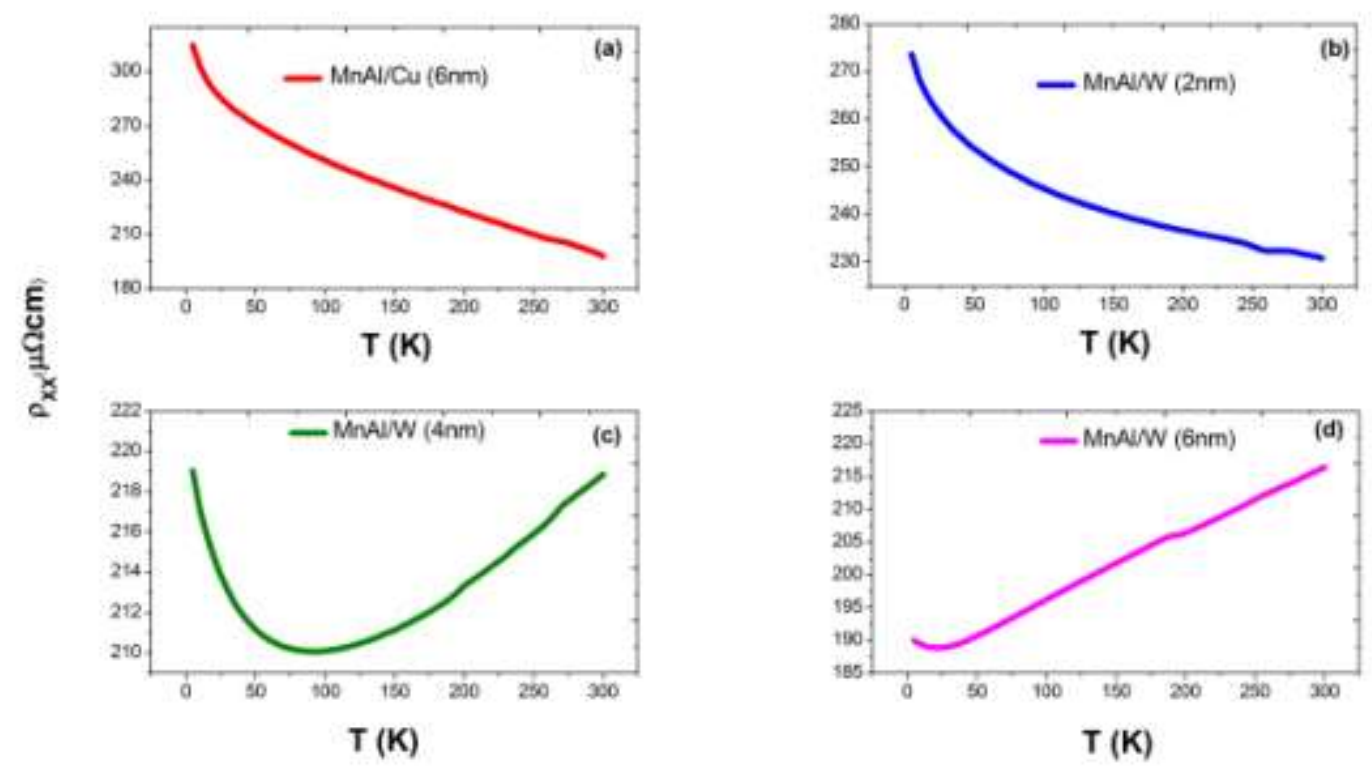

Fig. 2 The temperature dependence of the longitudinal resistivity $\rho_{\mathrm{XX}}$ for $\mathrm{MnAl} / \mathrm{Cu}(6 \mathrm{~nm})$ and $\mathrm{MnAl} / \mathrm{W}(\mathrm{t})$ films. 

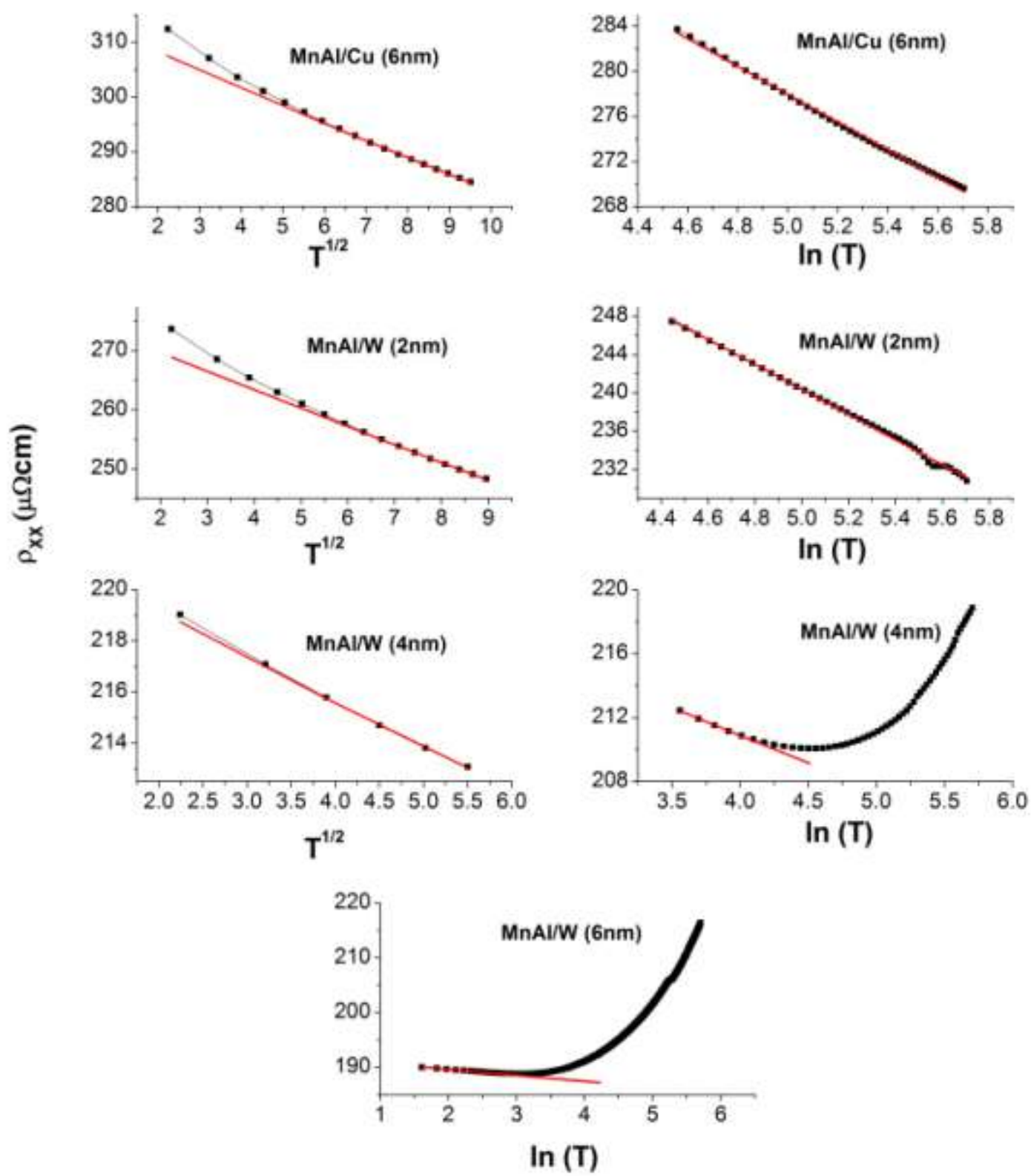

Fig. 3. The temperature dependence of the longitudinal resistivity $\rho_{\mathrm{XX}}$ for $\mathrm{MnAl} / \mathrm{Cu}$ $(6 \mathrm{~nm})$ and $\mathrm{MnAl} / \mathrm{W}(\mathrm{t})$. The red line is the fitted curve. 

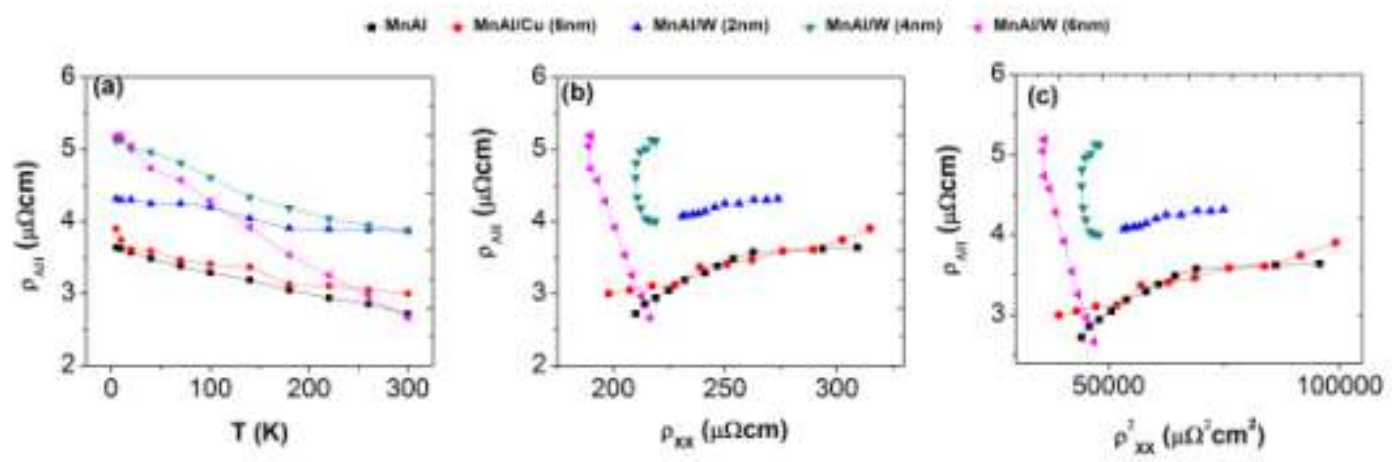

Fig. 4 (a) The temperature dependence of $\rho_{\mathrm{AH}}$ for the five samples. (b) $\rho_{\mathrm{AH}}$ as the function of $\rho_{\mathrm{XX}}$ for the five samples. (c) $\rho_{\mathrm{AH}}$ as the function of $\rho_{\mathrm{XX}}^{2}$ for the five samples.
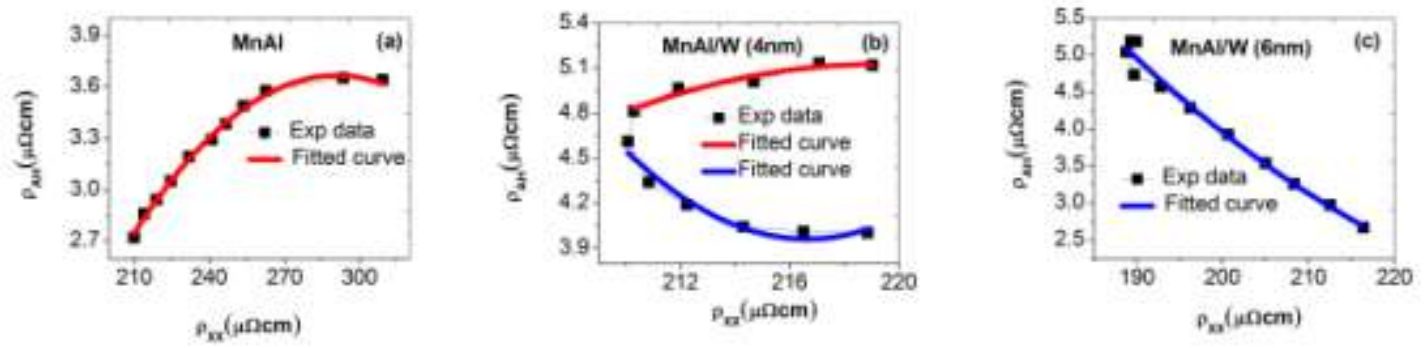

Fig. $5 \rho_{\mathrm{AH}}$ as the function of $\rho_{\mathrm{XX}}$ for the $\mathrm{MnAl}, \mathrm{MnAl} / \mathrm{W}(4 \mathrm{~nm})$ and $\mathrm{MnAl} / \mathrm{W}(6 \mathrm{~nm})$ films. 


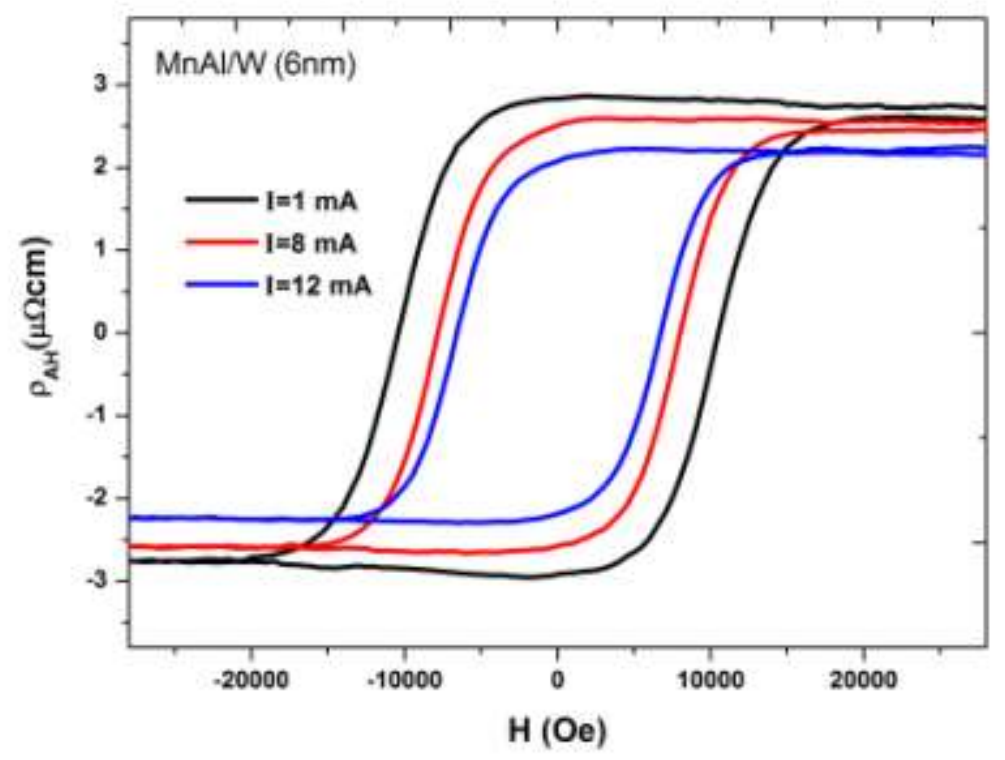

Fig. $6 \rho_{\mathrm{AH}}$ as the function of magnetic field with the input currents of 1,8 and $12 \mathrm{~mA}$ in $\mathrm{MnAl} / \mathrm{W}(6 \mathrm{~nm})$ film.

TABLE I. $T_{D}$ and $T_{K}$ for the five samples.

\begin{tabular}{cccccc}
\hline \hline & MnAl & MnAl/Cu(6nm) & MnAl/W(2nm) & MnAl/W(4nm) & MnAl/W(6nm) \\
\hline $\mathrm{T}_{\mathrm{D}}(\mathrm{K})$ & 40 & 40 & 30 & 10 & $\sim$ \\
$\mathrm{T}_{\mathrm{K}}(\mathrm{K})$ & 105 & 100 & 80 & 50 & 10 \\
\hline \hline
\end{tabular}

TABLE II. The AHE scaling coefficients for the five samples.

\begin{tabular}{ccccc}
\hline \hline & $\alpha$ & $\beta_{0}\left(\mu \Omega^{-1} \mathrm{~cm}^{-1}\right)$ & $\gamma\left(\mu \Omega^{-1} \mathrm{~cm}^{-1}\right)$ & $\beta_{1}\left(\mu \Omega^{-1} \mathrm{~cm}^{-1}\right)$ \\
\hline MnAl & $6.5 \mathrm{e}-3$ & $3.1 \mathrm{e}-5$ & $1.1 \mathrm{e}-4$ & $-1.4 \mathrm{e}-4$ \\
$\mathrm{MnAl} / \mathrm{Cu}(6 \mathrm{~nm})$ & $4.2 \mathrm{e}-3$ & $2.1 \mathrm{e}-4$ & $3.5 \mathrm{e}-3$ & $-2.2 \mathrm{e}-3$ \\
$\mathrm{MnAl} / \mathrm{W}(2 \mathrm{~nm})$ & $1.1 \mathrm{e}-2$ & $1.7 \mathrm{e}-4$ & $3.3 \mathrm{e}-4$ & $-2.6 \mathrm{e}-4$ \\
$\mathrm{MnAl} / \mathrm{W}(4 \mathrm{~nm})(\mathrm{T}<90 \mathrm{~K})$ & $2.1 \mathrm{e}-2$ & $5.4 \mathrm{e}-5$ & $3.2 \mathrm{e}-4$ & $-3.7 \mathrm{e}-3$ \\
$\mathrm{MnAl} / \mathrm{W}(4 \mathrm{~nm})(\mathrm{T}>90 \mathrm{~K})$ & $2.0 \mathrm{e}-2$ & $5.1 \mathrm{e}-5$ & $-8.9 \mathrm{e}-4$ & $1.3 \mathrm{e}-2$ \\
$\mathrm{MnAl} / \mathrm{W}(6 \mathrm{~nm})$ & $5.9 \mathrm{e}-2$ & $2.7 \mathrm{e}-5$ & $-2.7 \mathrm{e}-4$ & $8.6 \mathrm{e}-2$ \\
\hline \hline
\end{tabular}

